\title{
P07
}

\section{Acid Wormholing in Carbonate Reservoirs: Successes and Future Challenges}

\section{D.R. Guerillot* (Qatar Petroleum) \& V.R. Ambati (Qatar Petroleum)}

\section{SUMMARY}

Carbonate rock formation in petroleum reservoirs are highly heterogeneous in nature exhibiting complex porosity and permeability variations leading to irregular pore scale flow paths. Therefore, developing wormholes by acid stimulation in reservoir wells is favored for better injectivity and productivity of the wells. Determining and attaining a regime for wormhole generation in carbonates is very challenging as it depends on the variability of these petrophysical parameters. The objective of the present study is to stimulate the discussions on the following key questions:

1. How well-understood are the key mechanisms of the reactive-transport phenomena in acid wormholing?

2. What are the state-of-the-art numerical modelling techniques to capture this phenomena and associated commercial software's in the market?

3. How well-understood is this phenomena in the laboratory? And how valid are are the numerical predictions for laboratory experiments?

4. How deterministic is to characterize and predict acid wormholing in carbonate reservoirs? 